\title{
Influence of seeing effects on cloud model inversions
}

\author{
K. Tziotziou ${ }^{1}$, P. Heinzel ${ }^{2}$, and G. Tsiropoula ${ }^{1}$ \\ 1 National Observatory of Athens, Institute for Space Applications and Remote Sensing, 15236 Palea Penteli, Greece \\ e-mail: [kostas; georgia]@space.noa.gr \\ 2 Astronomical Institute, Academy of Sciences of the Czech Republic, CZ-25165 Ondřejov, Czech Republic \\ e-mail: pheinzel@asu.cas.cz
}

Received 9 May 2007 / Accepted 13 June 2007

\begin{abstract}
Context. Atmospheric seeing due to terrestrial atmospheric turbulence is a process that distorts solar ground-based observations. Aims. We study the influence of atmospheric seeing on $\mathrm{H} \alpha$ cloud model inversions.

Methods. We use Beckers' cloud model which assumes a constant source function to obtain physical parameters from observed quietSun $\mathrm{H} \alpha$ profiles, and profiles convolved with a Gaussian point spread function that simulates the effect of atmospheric seeing on solar observations.

Results. Our analysis indicates that atmospheric seeing does not affect the velocity determination, but does influence significantly the determination of the Doppler width when velocity gradients are present, and hence, the determination of the temperature, while the optical thickness and source function are also quantitatively affected, especially for high values of atmospheric seeing.

Conclusions. The effects of atmospheric seeing on cloud model inversions also depend on the actual size of the structures under study. For the presented observations, the derived cloud model parameter distributions do not change significantly for atmospheric seeing up to $\sim 0.5^{\prime \prime}$
\end{abstract}

Key words. methods: data analysis - Sun: general

\section{Introduction}

Apart from wavelength, space and time, solar line profiles depend on a multitude of parameters describing the solar environment they are formed in, such as chemical abundance, density, temperature, velocity, magnetic field, microturbulence, etc. When studying observed profiles, model atmospheres have to be assumed in order to restrict the number of these unknown physical parameters and to account for data noise. Procedures used for inferring these model parameters from observed profiles are known as "inversion techniques" (see Mein 2000, for a review).

Cloud models represent a class of solar models describing the transfer of radiation through cloud-like structures located above the solar surface, absorbing the radiation coming from below according to their optical thickness. Beckers (1964) introduced a simple inversion technique, known in the literature as "Beckers' cloud model", for inferring the cloud model parameters. Since then, several improvements of Beckers' cloud model have been suggested which concentrate on a more realistic form of the source function $S$, which in Beckers' cloud model is considered constant. We refer the reader to works by Mein \& Mein (1988), Gu et al. (1992, 1996), Mein et al. (1996), Tsiropoula et al. (1999), Tsiropoula (2000) and the review by Tziotziou (2007).

It is well known that solar/stellar observations from groundbased telescopes suffer from distortions. This effect is termed "atmospheric seeing" and is a statistical in nature process which adds noise to any solar/stellar ground-based observation. It is the result of turbulent motions in the Earth's atmosphere which produce random image distortion and blurring due to intermittent fluctuations of the refractive index. The quality of seeing is usually quantitatively measured either with a) the transverse coherent length $r_{0}$, which is the characteristic aperture diameter over which a plane wavefront is undistorted or $b$ ) the isoplanatic patch, which is the angular extent of an undistorted portion of the image. However, in practice, seeing in solar observations is defined by the size of the smallest visible structures in the fieldof-view.

In this paper we study how the effects of seeing influence the results obtained from the inversion of quiet-Sun $\mathrm{H} \alpha$ profiles, using the classical Beckers' cloud model.

\section{Beckers' cloud model}

The emerging line profile from a cloud-like structure is derived from the radiative transfer equation

$I(\Delta \lambda)=I_{0}(\Delta \lambda) \mathrm{e}^{-\tau(\Delta \lambda)}+\int_{0}^{\tau(\Delta \lambda)} S(t) \mathrm{e}^{-t(\Delta \lambda)} \mathrm{d} t$

which is the sum of the absorption by the cloud of the incident radiation $I_{0}(\Delta \lambda)$ from below (first term of the equation) and the emission by the cloud itself (second term of the equation). $I(\Delta \lambda)$ is the observed intensity, $\tau(\Delta \lambda)$ is the optical thickness and $S(t)$ the source function which is a function of optical depth along the cloud. Beckers (1964) assumed that the structure is completely separated from the underlying chromosphere and that the background intensity below the structure and in the surrounding atmosphere is the same and can be extrapolated from a neighboring region to the structure. He further assumed that the source function $S$, line-of-sight (LOS) velocity $v$, Doppler width $\Delta \lambda_{\mathrm{D}}$ and the absorption coefficient are constant along the LOS. Under these assumptions the radiative transfer equation has the formal solution

$I(\Delta \lambda)=I_{0}(\Delta \lambda) \mathrm{e}^{-\tau(\Delta \lambda)}+S\left[1-\mathrm{e}^{-\tau(\Delta \lambda)}\right]$ 

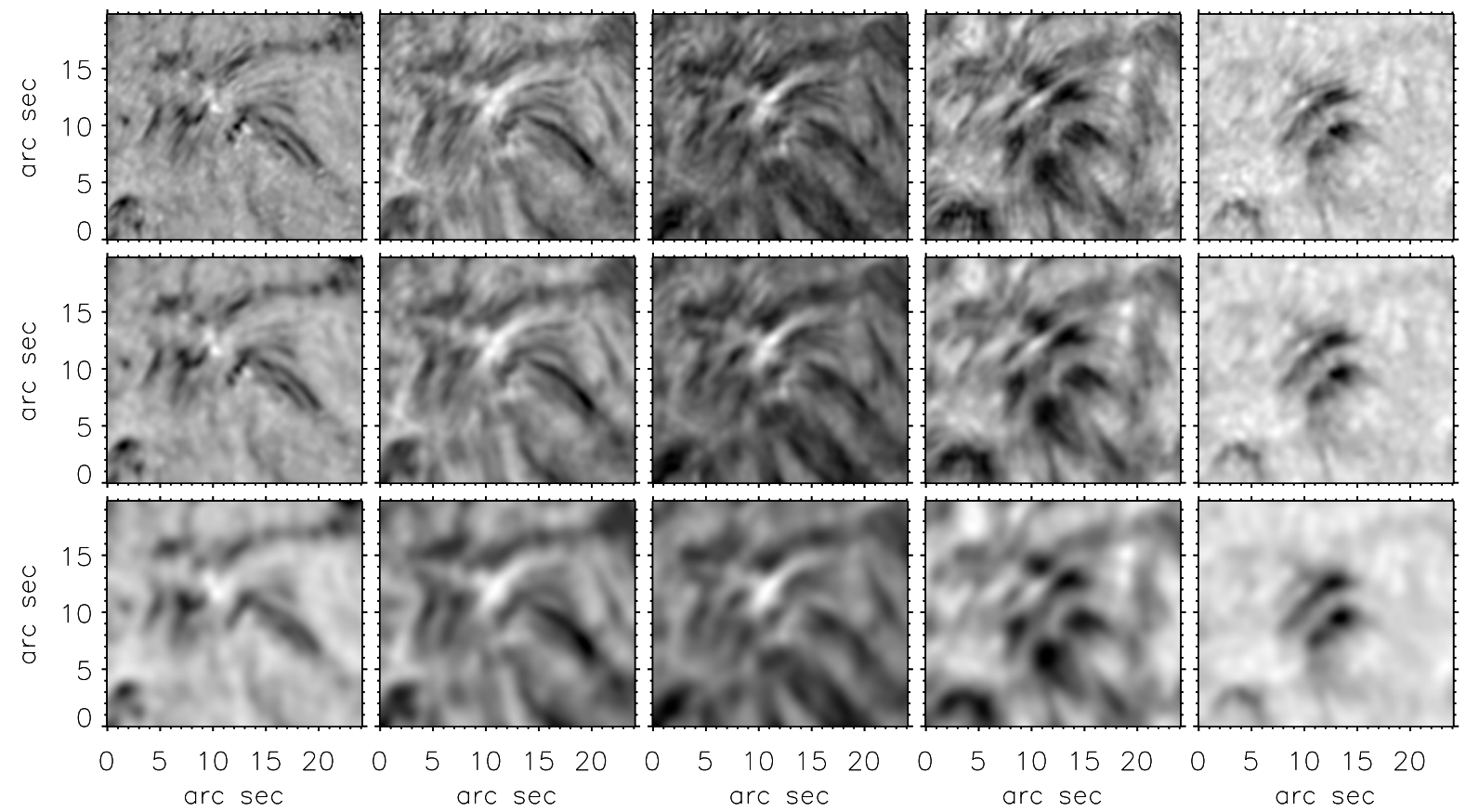

Fig. 1. $\mathrm{H} \alpha$ images of the rosette region at different wavelengths, starting from the blue wing (left column, $\mathrm{H} \alpha-0.7 \AA$ ) towards the red wing (right column, $\mathrm{H} \alpha+0.7 \AA$ ) with a wavelength step of $0.35 \AA$ (middle column shows the $\mathrm{H} \alpha$ line center), and for different seeing conditions. Top row shows the original observed images, middle row the same images with a simulated seeing of $\sim 0.5^{\prime \prime}$ and bottom row with a simulated seeing of $\sim 1.1^{\prime \prime}$.

and by defining the contrast profile $C(\Delta \lambda)$ as the ratio $[I(\Delta \lambda)-$ $\left.I_{0}(\Delta \lambda)\right] / I_{0}(\Delta \lambda)$, it can be rewritten as

$C(\Delta \lambda)=\left[\frac{S}{I_{0}(\Delta \lambda)}-1\right]\left[1-\mathrm{e}^{-\tau(\Delta \lambda)}\right]$.

For the optical thickness $\tau(\Delta \lambda)$ a Gaussian wavelength dependence is usually assumed

$\tau(\Delta \lambda)=\tau_{0} \mathrm{e}^{-\left(\frac{\Delta \lambda-\Delta \lambda_{\mathrm{I}}}{\Delta \lambda_{\mathrm{D}}}\right)^{2}}$,

where $\tau_{0}$ is the optical thickness at line center, $\Delta \lambda_{\mathrm{I}}=\lambda_{0} v / \mathrm{c}$ is the Doppler shift with $\lambda_{0}$ being the line center wavelength and $c$ the speed of light. The Doppler width $\Delta \lambda_{\mathrm{D}}$ depends on the temperature $T$ and the microturbulent velocity $\xi_{\mathrm{t}}$ as follows

$\Delta \lambda_{\mathrm{D}}=\frac{\lambda_{0}}{c} \sqrt{\frac{2 k T}{m}+\xi_{\mathrm{t}}^{2}}$,

where $m$ is the atom rest mass.

The four adjustable parameters of the model, assumed to be constant along the LOS, are the source function $S$, the Doppler width $\Delta \lambda_{\mathrm{D}}$, the optical thickness $\tau_{0}$ and the LOS velocity $v$. Beckers' cloud model works quite well for a large variety of optically thin or moderately thick structures and provides reasonable estimates for the physical parameters that describe them. Alissandrakis et al. (1990) discuss in detail the validity conditions of the model for different types of contrast profiles.

\section{Observations and data reduction}

The observations used in this study were obtained on October 14, 2005 with the Dutch Open Telescope (DOT) (Rutten et al. 2004). $\mathrm{H} \alpha$ image sequences were taken between 10:15 and 10:30 UT with DOT's tunable $\mathrm{H} \alpha$ Lyot filter at five wavelength positions: line center, $\pm 0.35 \AA$ and $\pm 0.7 \AA$. Speckle bursts of 100 frames each were taken at a rate of 6 frames $\mathrm{s}^{-1}$ (exposure time of $\leq 20 \mathrm{~ms}$ ) and the individual frames were speckle reconstructed (see Rutten et al. 2004 for details), yielding one reconstructed image per burst. All individual images were carefully aligned in time, giving a time sequence consisting of 26 high resolution (average $r_{\mathrm{o}}=7 \mathrm{~cm}$, spatial resolution $\sim 0.2^{\prime \prime}$ ) frames of size 1106 pixels $\times 838$ pixels with $0.071^{\prime \prime} /$ pixel and a $35 \mathrm{~s}$ cadence. We will consider the seeing in the observed images as zero, and we will use these original images to simulate atmospheric seeing effects on cloud model results.

Only the speckle reconstructed time series is available for our study and not the individual burst images. Hence a) no verification of the correctness of the alignment, especially between the blue and red wing wavelengths of the line, is possible and b) no comparison between the original burst images of variable seeing with the simulated seeing images can be performed for checking both the seeing simulation and also possible artifacts introduced by the speckle reconstruction.

The observed region is a solar disk center quiet-Sun area (N06W00) containing several mottles. We however, for computational reasons, applied Beckers' cloud model to only a $24^{\prime \prime} \times 19.8^{\prime \prime}$ part of the original images containing a rosette of mottles (top row of Fig. 1) to derive the physical parameters which describe the mottles contained in the region. The cloud model equations were solved with an iterative least-square procedure for non-linear functions (Tziotziou et al. 2003). The background intensity at each wavelength was taken as the average intensity at the same wavelength of the selected region. Although this definition of the background intensity may not be absolutely correct, since it does not necessarily correspond to a quiet-Sun region and would thus affect quantitatively the physical parameters obtained, it does not affect our statistical analysis because the same background definition was used for all original 
and simulated images. Only $\mathrm{H} \alpha$ profiles of mottles that have a contrast at line center lower than zero have been considered; this means that we are considering only structures in absorption which appear dark compared to the background intensity. The inversion of an observed profile is considered successful when the relative decrease of $\chi^{2}$, which measures the goodness of the fit of the observed profile, is less than $10^{-10}$ in one iteration and all obtained cloud model parameters are physically correct (positive values for optical thickness, source function and Doppler width and reasonable values of Doppler width). The derived $\chi^{2}$ distributions peak at $\sim 3 \times 10^{-4}$ and have a mean of $\sim 7 \times 10^{-4}$.

\section{Simulating the seeing}

Seeing effects on solar observations can be simulated by convolving the observations with a suitable point spread function (PSF) representing these effects. In principle PSFs can be complicated functions also containing non-Gaussian components. However, for reasons of simplicity, it can be assumed to be a two-dimensional Gaussian function with its FWHM representing quantitatively the atmospheric seeing. We have used a twodimensional 50 pixel $\times 50$ pixel Gaussian PSF for convolving the original images with a variable appropriate $F W H M$ (from 2 to 15 pixels, corresponding to an atmospheric seeing of $0.15^{\prime \prime}$ to $\left.1.07^{\prime \prime}\right)$ and variable amplitude to ensure that the total volume integral of the PSF is equal to one. We assume that the noise introduced by the optical system (e.g. scattered light) is independent of the seeing conditions and thus can be neglected in our statistical analysis. The second and third rows of Fig. 1 show examples of observed images in all five wavelengths (first row) with a simulated seeing of $\sim 0.5^{\prime \prime}$ and $\sim 1.1^{\prime \prime}$ respectively. We see that several thin structures either completely vanish when the seeing increases or merge with other thin structures in their vicinity to form visually larger thick structures, while their contrast becomes smoother and less detailed. It is also worth noticing that granulation completely disappears for high atmospheric seeing.

\section{Results}

We have applied the cloud model to the original observations (which consist of five-wavelength $\mathrm{H} \alpha$ profiles at each pixel of the two-dimensional image) and to the corresponding observations with simulated seeing effects in order to study the influence of seeing on the obtained physical parameters of mottles.

Figure 2 shows the distributions of the calculated cloud model parameters derived for the $\mathrm{H} \alpha$ observations of Fig. 1 with no seeing effects (solid thick line) and for simulated seeing of $\sim 0.5^{\prime \prime}$ (solid thin line) and $\sim 1.1^{\prime \prime}$ (dotted line). We see that although the distributions look similar there are obvious qualitative and sometimes significant quantitative differences especially for the distributions of Doppler width and optical thickness. Distributions of the parameters obtained from the observations at each particular time of the time sequence show the same behaviour. Differences in the Doppler width distribution raise either by smoothing over pixels with different Doppler widths or, mainly, from the presence of velocity gradients. In the latter case, i.e. when the structures under study exhibit velocity gradients, profiles corresponding to neighboring pixels will be shifted differentially and hence smoothing by atmospheric seeing will affect the profile width. On the other hand, positive or negative flows have as a result the asymmetric broadening of the line profile, giving rise to a blue or red asymmetry respectively, but the effect in both cases on the line width and hence the
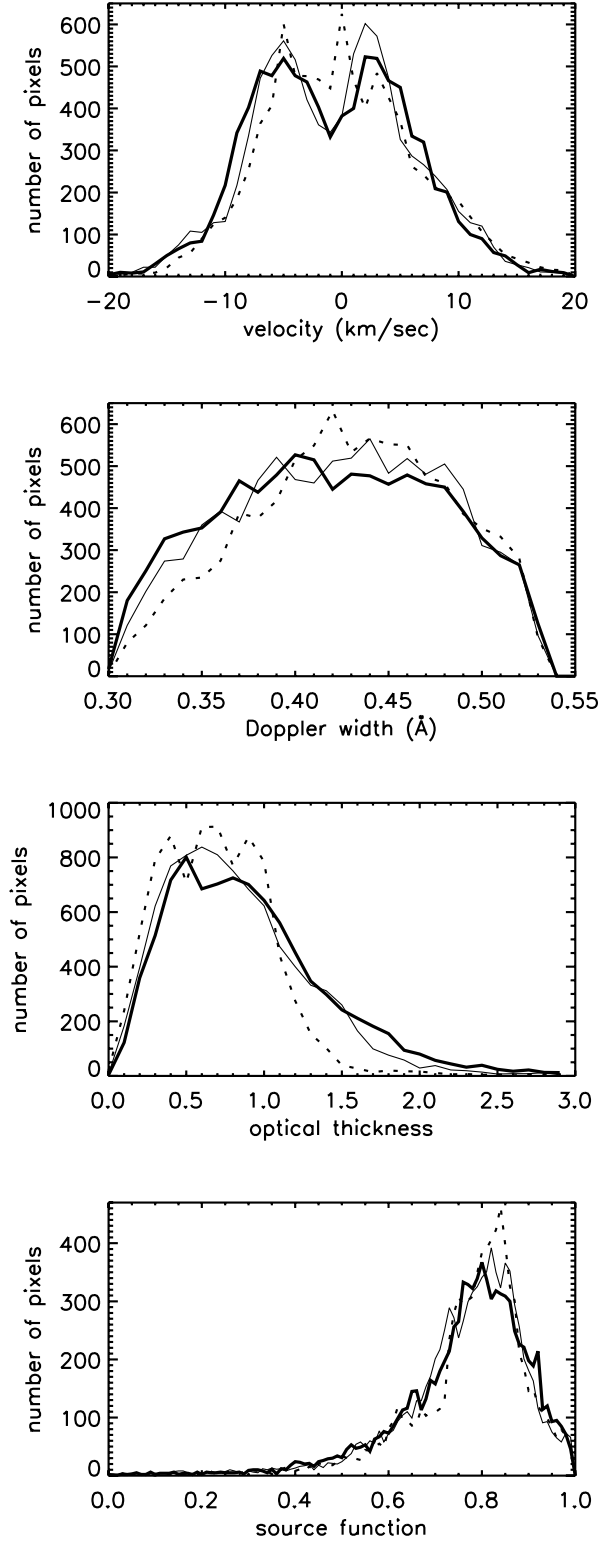

Fig. 2. Distributions of the calculated cloud model parameters derived from the original $\mathrm{H} \alpha$ observations (solid thick line) and for the same observations with simulated seeing of $\sim 0.5^{\prime \prime}$ (solid thin line) and $\sim 1.1^{\prime \prime}$ (dotted line). The source function is taken relative to the background line center intensity. The $\mathrm{H} \alpha$ observations are those presented in Fig. 1 and correspond to a time of observation of $525 \mathrm{~s}$ in Fig. 3.

Doppler width value is similar. It has been clearly demonstrated by Tsiropoula et al. (1993) and Tziotziou et al. (2003) that there are significant absolute velocity gradients, both positive and negative, along the main axis of mottles and also between mottles and the surrounding background. Hence, smoothing introduced by atmospheric seeing will result to different Doppler width distributions. The fact that the Doppler width distribution of Fig. 2, especially for a seeing of $\sim 1.1^{\prime \prime}$, shows a decrease of the number of pixels with Doppler widths less than $0.4 \AA$ and an increase of the number of pixels with Doppler widths higher than $0.4 \AA$ compared to the original distribution could be explained as follows: smoothing due to atmospheric seeing of profiles corresponding to the original Doppler width distribution, which reflects the presence of velocity gradients, will broaden the profiles and lead to a shift of the distribution towards larger 


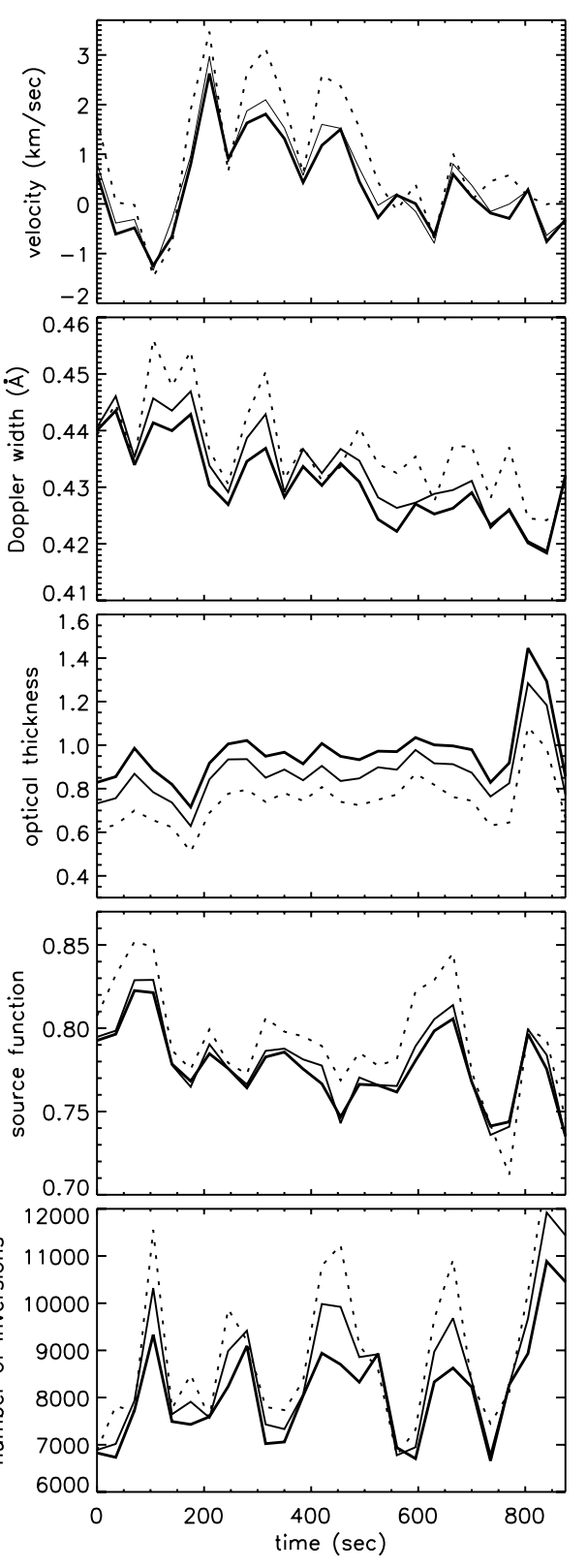

Fig. 3. Mean values of velocity (top row), Doppler width (second row), optical thickness (third row), source function (fourth row), as well as the number of successful inversions (bottom row) as a function of time and for different values of seeing for the entire time sequence. Thick solid line corresponds to the original $\mathrm{H} \alpha$ observations, thin solid line to the observations with simulated seeing of $\sim 0.5^{\prime \prime}$ and dotted line to the images with simulated seeing of $\sim 1.1^{\prime \prime}$. The source function is taken relative to the background line center intensity.

Doppler widths, with narrow profiles transforming to broader ones and thus getting less number of pixels below some limit and more above it. Atmospheric seeing influences also the determination of optical thickness and source function. With better seeing the contrast is better and structures are darker as the images of Fig. 1 clearly show. When the seeing is worse, the visual contrast $I(\Delta \lambda)-I_{0}(\Delta \lambda)$ lowers and this looks like lower opacity or higher source function leading to the derived distributions of smaller optical thickness and higher source function.

The effect of atmospheric seeing on the parameter distributions can be better seen in Fig. 3, which shows the mean values of the derived parameters over the entire field of view, as well as the number of successful inversions, as a function of time for the original observations (thick solid line) and for observations with different values of simulated seeing (thin solid line and dotted line for simulated seeing of $\sim 0.5^{\prime \prime}$ and $\sim 1.1^{\prime \prime}$ respectively). We see that the values of velocity, Doppler width and source function increase when the atmospheric seeing increases for all observations of the time sequence, while the optical thickness decreases for increasing seeing values. The number of successful inversions also increases substantially with increasing seeing as a result of the smoothing that atmospheric seeing introduces to an observed image. The observed increase of mean velocity and Doppler width with seeing reflects the presence of velocity gradients due to the nature of the structures under study (mottles), which are jet-like structures. Among the four parameters, velocity seems to be the least affected, at least for reasonable values of seeing, which is in accordance to the general belief in the literature that the velocity is the best defined cloud model parameter. This is because: a) its determination depends on the shape of the contrast profile which is not that much affected by seeing conditions, that have a similar qualitative influence on both the observed and the background profiles and $b$ ) the smooth variation of velocity in the structures under study. There is a quite substantial decrease of the optical thickness and a considerable increase of the source function, as also the respective distributions of Fig. 2 clearly indicate, in accordance with the above discussion about the effect of atmospheric seeing on these two cloud model parameters. It is interesting to note the anti-correlation between the Doppler width and the optical thickness, which reflects the fact that the line-center thickness gets lower for broader profiles. There also appear to be oscillations present in all panels of Fig. 3. The oscillations in the time variation of the mean values of the four cloud parameters seem to reflect the time variation of the background intensity (not presented here). The 3-min oscillation observed in the time variation of the number of inversions is somewhat spurious, since it is reminiscent of the well known 3-min chromospheric oscillation. It could however be coincidental.

Figure 4 shows scatter plots of the derived cloud model parameters between the original $\mathrm{H} \alpha$ observations (shown in Fig. 1) and those derived from observations with simulated seeing $\sim 0.5^{\prime \prime}$ (top row) $\sim 1.1^{\prime \prime}$ (bottom row). A similar behavior is observed in the scatter plots between all observations of the time sequence and their corresponding observations with simulated increasing seeing. The best correlation, when seeing is increasing, still remains for the derived velocity values, as also the parameter distributions of Fig. 2 and the mean values time behavior in Fig. 3 have indicated. The parameter which is mostly influenced by increasing seeing is the Doppler width, due to the presence of velocity gradients. It is also worth noticing that both velocity and Doppler width scatter plots are symmetrical to the diagonal (dotted line); this is also true partly for the optical thickness and source function distributions, except for high/low values respectively of the original distributions. This is due to the smoothing introduced by seeing which has as a result that two or more parameter values of neighboring pixels, represented initially as points on the diagonal, will now shift vertically in the scatter plot as a result of the new smoothed value. Hence, for one value now on the vertical axis we get two or more "symmetrical" values with respect to the diagonal on the horizontal axis. The effect is more pronounced for Doppler width since this is the parameter mostly affected by seeing conditions. Increasing seeing also results, as expected, in lower values of the optical thickness and higher values of the source function with the highest divergence observed for higher values of the original optical thickness and lower values of the original source function 

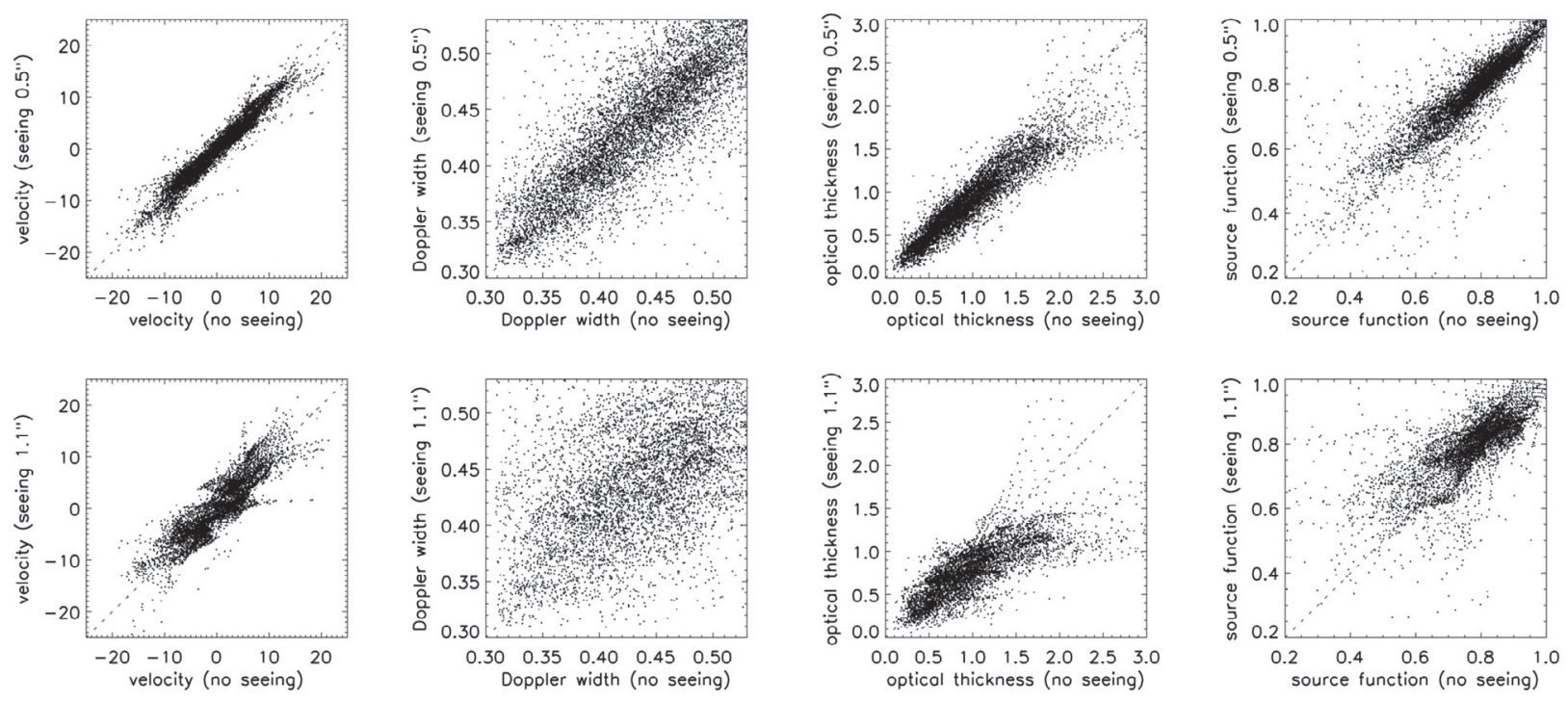

Fig. 4. Scatter plots between the derived cloud model parameters for the original H $\alpha$ observations shown in the top row of Fig. 1 and their corresponding observations with simulated seeing of $\sim 0.5^{\prime \prime}$ (top row) $\sim 1.1^{\prime \prime}$ (bottom row) which are shown in the middle and bottom row of Fig. 1 respectively. The source function is taken relative to the background line center intensity.

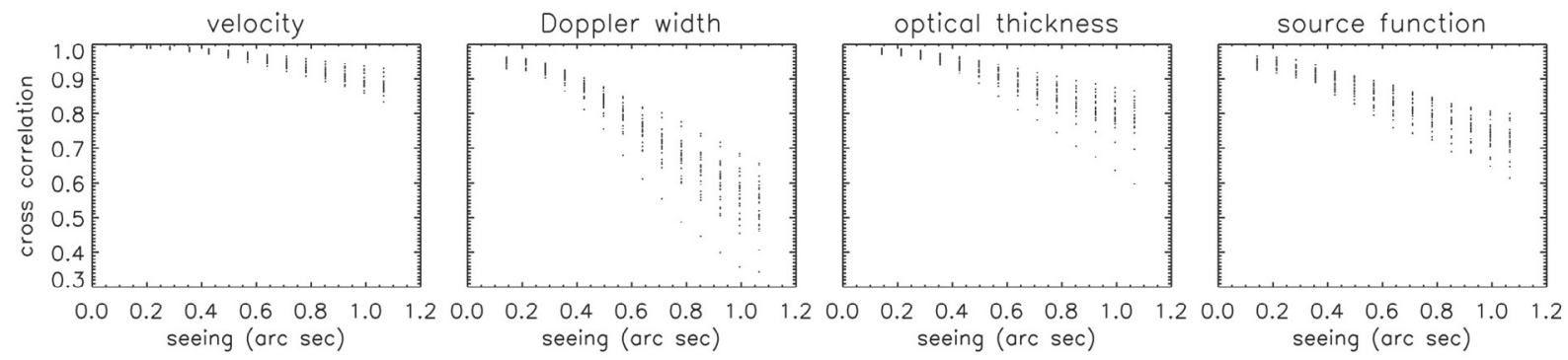

Fig. 5. Cross correlations for the four cloud model parameters derived for images of the time sequence and their corresponding images with simulated, variable seeing as a function of atmospheric seeing. A value of 1 shows complete correlation, while a value of 0 shows a lack of correlation.

which are mostly affected by smoothing introduced by seeing conditions. These results have also been clearly reflected in the obvious changes of the corresponding distributions presented in Fig. 2.

A better, more comprehensive and "quantitative", way of representing the aforementioned influence of atmospheric seeing to observations is to calculate the cross-correlation between parameter values derived for each observation of the time sequence and the respective parameter values for the corresponding observations with simulated, variable seeing. The cross-correlation is a quantity between -1 and 1 , where -1 represents a complete anti-correlation between the two samples, 0 a lack of correlation and 1 a complete correlation. Figure 5 shows the computed cross correlations for the four cloud model parameters as a function of atmospheric seeing for all images of the time sequence (Fig. 4 shows what the used scattered plots for the computed cross-correlations look like, but only for one image of the time sequence). We notice that the cross-correlation of the velocities does not change substantially with increasing atmospheric seeing, in accordance with all previous results of this study, while the behavior of the optical thickness and the source function with increasing seeing is almost similar, i.e. they decrease with the same rate. This is due to the fact that the quantitative definition of both the optical thickness and the source function in the cloud model inversion is strongly coupled with the line center intensity. Hence, any atmospheric seeing effects, which will affect the value of this intensity, will have similar but opposite effects on both quantities, i.e. an increase of the source function and a decrease of the optical thickness, an effect clearly seen in the scatter plots of Fig. 4. What is noticeable, but yet expected from the previous discussion of the results, is the substantial decrease of the cross-correlation for the Doppler width with increasing atmospheric seeing when velocity gradients are present. This shows that atmospheric seeing strongly affects the determination of temperature from the Doppler width according to Eq. (5) when a constant value of microturbulence is assumed. It also highlights that higher values of microturbulence have to be assumed for the determination of reasonable values for the temperature with increasing atmospheric seeing, since the latter, although a different in nature process than microturbulence which introduces Doppler effects on the profile during its formation, has a similar effect on the width of the profiles in the presence of velocity gradients.

The vertical spread of the cross-correlation curve is mostly due to temporal differences in the background intensity. Since the background intensity has been defined as the average of the whole region under study, there are slight differences in the acquired background intensity from image to image due to the presence of greater or fewer dark structures.

From Fig. 5 one can easily deduce what the effect of atmospheric seeing will be on time slice images which show time variations of parameters along one-dimensional cuts which are 
commonly used in literature for the study of quiet-Sun structures with cloud models (e.g. Tziotziou et al. 2003). There will hardly be any quantitative differences for the velocity behavior with increasing seeing, while the optical thickness and source function behavior is going to be qualitatively similar, but with quantitative differences especially for high values of atmospheric seeing. Finally, the Doppler width behavior is the only one that can show significant qualitative variations with increasing values of atmospheric seeing when velocity gradients are present in the observations.

\section{Discussion and conclusions}

We have studied the influence of atmospheric seeing on cloud model inversions performed with the classic Beckers' cloud model which assumes constant values for all four parameters that describe cloud-like solar structures. Our analysis has indicated that atmospheric seeing does not greatly affect the velocity determination, but does influence significantly the determination of the Doppler width when velocity gradients are present and, hence, the determination of the temperature. The optical thickness and source function, which are strongly coupled with the line center intensity, are also quantitatively affected, especially for high values of atmospheric seeing, but qualitatively the parameter distributions for these observations do not change significantly for atmospheric seeing of the order of $0.5^{\prime \prime}$. It is also worth mentioning that Mein et al. (1996) came to conclusions similar to ours for the velocity and optical thickness behavior when a Gaussian noise, that corresponds approximately to a relative fluctuation of $3 \%$ at the line center and $1 \%$ at $\pm 0.6 \AA$, was introduced to their observed $\mathrm{H} \alpha$ data.

Our analysis has indicated once more the important role of the background in the determination of physical parameters. The correct choice of the background intensity is crucial for the accurate determination of the physical parameters. However, the background choice used in our analysis, i.e. by taking the image average at each wavelength, does not affect the conclusions of our analysis, since the same determination has been used for all original and simulated images of the time sequence. It is obvious, of course, that the choice of the image average in conjunction with the choice of a Gaussian PSF, that has an integral of one for simulating atmospheric seeing effects and thus introduces a uniform smoothing that does not alter significantly the average intensity, will have a quantitatively different result than using only a small part of the image for determining the background, where smooth effects could play an extra role. However, the latter would be too difficult to interpret because it would be hard to disentangle the role of atmospheric seeing from the role of the background intensity in the determination of the cloud model parameters. A test inversion, where the average intensity of the whole 1106 pixels $\times 838$ pixels observed image at each wavelength was taken as the background intensity at the same wavelength, has given similar results on the influence of seeing on cloud model inversions.
The choice of a Gaussian PSF for simulating atmospheric seeing is also a limitation in our analysis. In principle, atmospheric seeing and also the telescope's optical system could introduce a much more complicated function containing nonGaussian components. The Gaussian choice, however, represents the best and simplest to comprehend and interpret function for atmospheric seeing. We believe that more complicated forms would not alter significantly - at least qualitatively - the conclusions of our analysis.

One may also argue that the choice of Beckers' cloud model for cloud model inversions poses another serious limitation in our analysis. It has however been extensively indicated in the literature that inversion results do not differ significantly especially for optically thin structures when different variants of the cloud model are used (i.e. parabolic source function, source function depending on line-center optical thickness etc.). For examples, we refer the reader to the studies by Alissandrakis et al. (1990), Mein et al. (1996), Heinzel et al. (1999) and the review by Tziotziou (2007) for an overview of the influence of cloud model methods on obtained results. Hence, the conclusions of our analysis would not alter significantly when choosing a different cloud inversion method.

Finally, we note that the effects of atmospheric seeing on cloud model inversions also depend on the actual size of the structures under study. This size is limited by the spatial resolution of the images and depends also on the instrument used for the observations. It is obvious that for structures which are geometrically extended, with sizes comparable to atmospheric seeing, the effect of seeing will be less remarkable compared to very narrow ones such as mottles which are the subject of this study.

Acknowledgements. We thank P. Mein for constructive discussions and comments. This work has been partly supported by a Greek-Czech programme of cooperation.

\section{References}

Alissandrakis, C. E., Tsiropoula, G., \& Mein, P. 1990, A\&A, 230, 200

Beckers, J. M. 1964, A study of the fine structures in the solar chromosphere, Ph.D. Thesis, Utrecht

Gu, X. M., Lin, J., Luan, T., \& Schmieder, B. 1992, A\&A, 259, 649

Gu, X. M., Lin, J., Li, K. J., \& Dun, J. P. 1996, A\&ASS, 240, 263

Heinzel, P., Mein, N., \& Mein, P. 1999, A\&A, 346, 322

Mein, P. 2000, in Advances in Solar Research at Eclipses from Ground and from Space, ed. J.-P. Zahn \& M. Stavinschi, NATO ASIC Proc., 558, 221

Mein, P., \& Mein, N. 1988, A\&A, 203, 162

Mein, N., Mein, P., Heinzel, P., et al. 1996, A\&A, 309, 275

Rutten, R. J., Hammerschlag, R. H., Bettonvil, F. C. M., Sütterlin, P., \& de Wijn, A. G. 2004, A\&A, 413, 1183

Tsiropoula, G. 2000, New Astron., 5, 1

Tsiropoula, G., Alissandrakis, C. E., \& Schmieder, B. 1993, A\&A, 271, 574

Tsiropoula, G., Madi, C., \& Schmieder, B. 1999, Sol. Phys., 187, 11

Tziotziou, K. 2007, in Coimbra Solar Physics Meeting on The Physics of Chromospheric Plasmas, ed. P. Heinzel, I. Dorotovič, \& R. J. Rutten, ASP Conf. Ser., 368, 217

Tziotziou, K., Tsiropoula, G., \& Mein, P. 2003, A\&A, 402, 361 